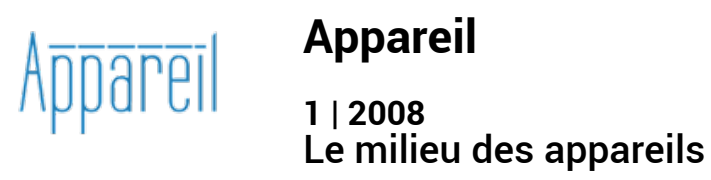

\title{
Architectures de la transparence
}

\section{Emmanuel Alloa}

\section{OpenEdition}

Journals

Édition électronique

URL : http://journals.openedition.org/appareil/138

DOI : 10.4000/appareil. 138

ISSN : 2101-0714

\section{Éditeur}

MSH Paris Nord

\section{Référence électronique}

Emmanuel Alloa, «Architectures de la transparence », Appareil [En ligne], 1 | 2008, mis en ligne le 21 février 2008, consulté le 30 juillet 2020. URL : http://journals.openedition.org/appareil/138 ; DOI : https://doi.org/10.4000/appareil.138

Ce document a été généré automatiquement le 30 juillet 2020.

\section{(c) (i) (2) $\Theta$}

Appareil est mis à disposition selon les termes de la Licence Creative Commons Attribution - Pas d'Utilisation Commerciale - Pas de Modification 4.0 International. 


\title{
Architectures de la transparence
}

\author{
Emmanuel Alloa
}

\section{Transparences}

«Si droite est ma vision, si pure ma sensation, si maladroitement complète ma connaissance, et si déliée, si nette ma représentation, et ma science si achevée que je me pénètre depuis l'extrémité du monde jusqu'à ma parole silencieuse; et de l'informe chose qu'on désire se levant, le long de fibres connues et de centres ordonnés, je me suis, je me réponds, je me reflète et me répercute, je frémis à l'infini des miroirs - je suis de verre. »

Paul Valéry L'homme de verre (Monsieur Teste-1903)

Dans ce passage de L'homme de Verre, poème en prose recueilli dans le Log-Book de Monsieur Teste, Paul Valéry réitère à sa façon - en ventriloque malicieux du célèbre gentilhomme dont «la bêtise n'est pas [le] fort »- ce rêve immémorial de l'orthotheron blepoi, cette "vision droite » à laquelle fait allusion la République de Platon et sur lequel Descartes fondera son axiologie du Discours de la Méthode. Une vision droite donc, pénétrante, qui ne connaîtrait aucun obstacle et qui retrouve son propre reflet jusque dans les recoins les plus éloignés du monde. Des reflets qui ne sauraient être rien d'autre que la série infinie de reflets d'autres reflets, car rien ne pourrait habiter cet homme de verre qu'un être éternellement réfléchissant.

2 Qui est Teste ? Témoin sans doute d'une époque révolue, individu (pour le dire avec Walter Benjamin) «qui, sur le point de franchir le seuil de la disparition historique, ombre déjà, répond une dernière fois à l'appel de son nom, avant de plonger là où personne ne l'atteint plus» mais aussi - Walter Benjamin y est particulièrement sensible - l'avant-coureur d'un monde dont ses contemporains ne distinguent pas encore les lignes directrices effectives ${ }^{1}$. Car loin d'être seulement l'apanage d'un sujet à la limite de la dissolution physique, l'idéal de la transparence peut être considéré Anthony Vidler l'a montré dans The Architectural Uncanny - comme le mythe traversant et s'incorporant dans la modernité tout entière ${ }^{2}$. De tous les idéaux des Lumières qui fonderont les principes du moderne, la transparence est assurément à la fois la plus ancienne, mais à la fois aussi celle qui survit à l'érosion morale de toutes les autres pour 
devenir la valeur consensuelle et inquestionnée de notre temps. La transparence du soi à soi, du soi et de son savoir, du soi et de l'autre et de la société à elle-même, sera passée brièvement par sa phase utopique ( La transparence, pensait-t-on, éradiquerait tous les domaines du mythe, la suspicion, la tyrannie, et - avant tout - l'irrationnel $\left.{ }^{3} »\right)$ pour trouver à notre époque, bouturant la modernité classique, dans la bureaucratisation anhistorique son sacre définitif. À la différence du frémissement de verre chez Monsieur Teste, la transparence se doit, à l'ère médiatico-politique, d'être montrée, exhibée, affichée en public, l'affiche de la transparence (son " display ») garantissant l'absence de mainmise étrangère.

3 Avant de pouvoir analyser les avatars contemporains de cette idéologie de la transparence bureaucratique - qui, comme toute idéologie, se défend d'en être une (1.), on choisira de faire le détour par quelques-unes des ses cristallisations antérieures. Suivant l'idée de Vidler selon laquelle le mythe de la transparence se concrétise avant tout dans l'idée d' "une transparence universelle des matériaux de construction ${ }^{4} »$, on prendra pour stations historiques l'architecture de la lumière à l'époque gothique et son reflet dans la structure cristalline de la dialectique scolastique (2.), l'avancée technologique du fer et du verre au début du XIX ${ }^{e}$ siècle ensuite, rendant possible les grandes expositions universelles et l'utopie de la circulation sans entraves (3.), les rêveries utopiques enfin autour d'une "civilisation de verre" avant et après la Première guerre mondiale, antécédents souvent oubliés du modernisme fonctionnaliste (4.) servant à son tour de caution aux nouvelles architectures de la transparence dès les années 1960 , où se forge un nouveau langage international de la lubrification des services, dominant aujourd'hui.

\section{Transparences verticales}

"Lumineux est le noble édifice que la clarté envahit »

Abbé Suger de Saint-Denis

L'avènement $\mathrm{du}$ gothique au $\mathrm{XII}^{\mathrm{e}}$ siècle constitue, sans nul doute, la première concrétisation effective d'un espace transparent entre ce qui était jusque là distinct. L'idée, remontant à l'époque théodosienne au moins, selon laquelle dans la basilique chrétienne se matérialise déjà la Jérusalem Céleste dépassera dès à présent le stade de l'idée: les techniques architecturales nouvelles permettent l'érection de nombreuses tours et les constructions surmontant les culées extérieures évoquent à elles seules les visions eschatologiques de saint Jean; mais, plus encore, c'est à l'intérieur, dans l'espace même de l'Église, que la communauté des fidèles peut avoir un avant-goût de la Cité Céleste décrite dans le Quatrième Évangile: l'architecture en voûtes d'ogive permet un allègement spectaculaire de la masse, baignant ainsi dans une lumière unique les espaces qui restaient jusque là séparés par les travées. L'expérience vécue par la communauté d'une lumière divine pénétrant par les vitraux est celle d'une " communion » dans et par la grâce qui ne connaît désormais plus d'obstacles. Pour la première fois, cette communauté dans l'« espace-lumière » (L. Grodecki) s'effectue dans la nouvelle basilique gothique de Saint-Denis, érigée par son abbé Suger (1081-1151). La nouvelle bâtisse qui se construit au même endroit que l'ancienne église du roi Dagobert ne garde plus aucun élément de sa structure originale - Erwin Panofsky affirmera que le geste architectural de Suger n'est pas moins radical que si le président des États-Unis avait demandé à Frank Lloyd Wright de redessiner la Maison Blanche ${ }^{5}$. 


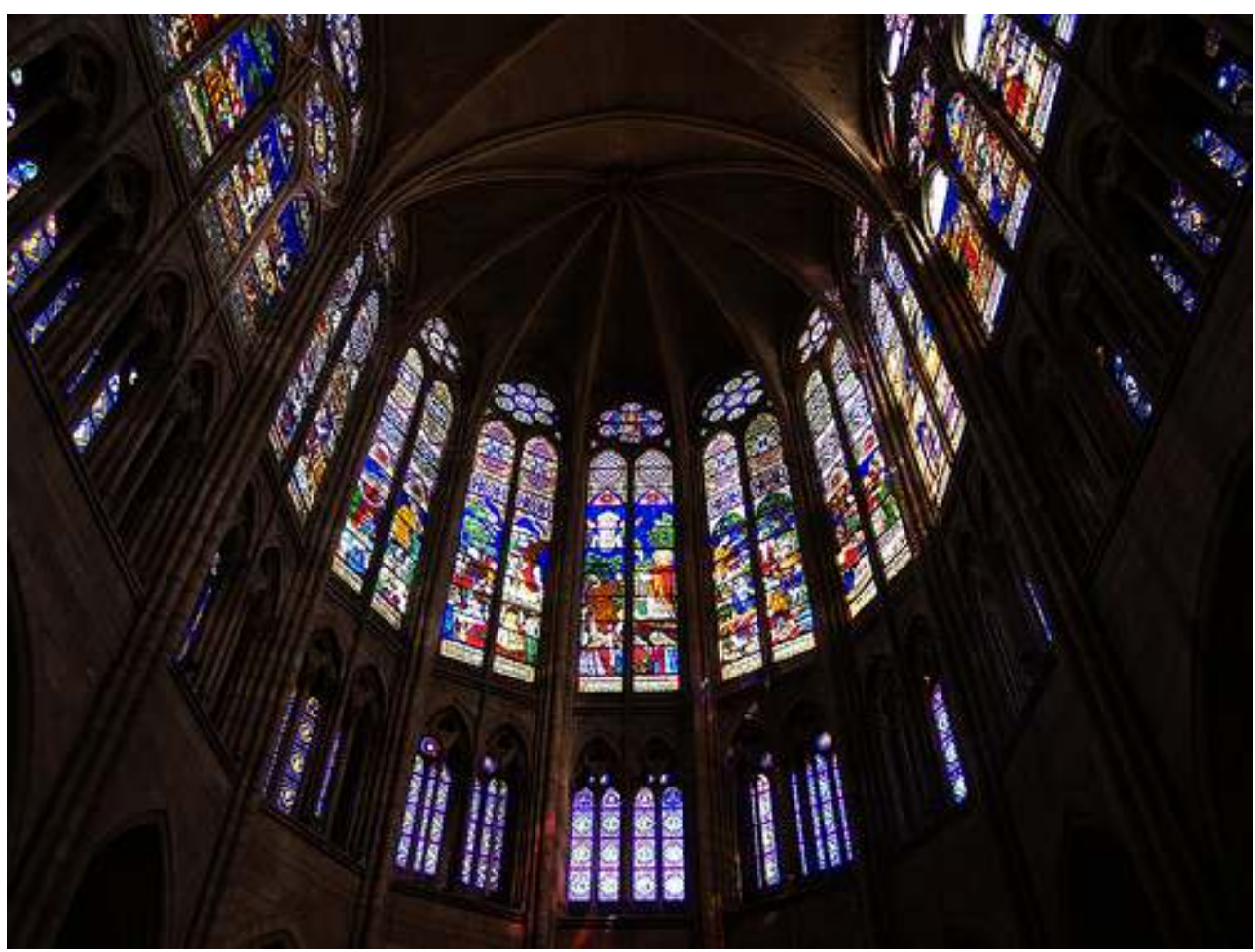

Andrew Gainer, Basilique de Saint-Denis, Vitraux, 2006

(C) Courtesy of Andrew Gainer

5 Suger aura, en effet, fait abattre l'ancienne abside et la façade ouest abritant la sépulture de Pépin le Bref, il aura fait ériger un nouveau narthex ainsi qu'un nouveau chœur et aura ordonné de raser les restes de l'ancienne basilique reprises dans la nef. En brisant l'opacité des murs carolingiens, Suger ouvre l'espace ecclésial pour y laisser pénétrer la lumière qu'il considère déjà comme une émanation divine. Quand les travaux seront achevés, les séparations entre les nefs seront abolies et la cathédrale deviendra un unique navire lumineux. Dans le chœur, inauguré en 1144, Suger fait graver le versiculus (inscription versifiée) suivant : «Parce que la nouvelle partie rejoint l'antérieure/ la salle éclairée en son centre resplendit/ Car ce qui se fond avec le clair très clair rayonne/ et parce qu'une nouvelle lumière [lux nova] la traverse, l'œuvre noble reluit $/{ }^{\circ} »$. Cette lux nova représente à la fois les rayons de lumière sensibles (lumina) et la vraie lumière, suprasensible, de la révélation (verum lumen) auxquels ils participent. Par un procédé anagogique, la lumière physique élève le spectateur vers la luminosité divine, établissant ainsi une continuité visuelle (lux continua) entre monde et hors-monde. On trouve dans l'écrit De consecratione la formulation du principe de lumière continue : "en agrandissant élégamment les chapelles environnantes, toute l'église resplendira à travers les vitres lumineuses d'une lumière merveilleuse et continue quand la beauté de l'intérieur en sera éclairée ${ }^{7}$ ».

6 Dans une interprétation qui fit date, Erwin Panofsky proposa de lire l'architecture de Saint-Denis comme symptôme d'une esthétique de la transparence ayant pour équivalent la structure argumentative des traités philosophiques de la scolastique ${ }^{8}$. Le principe de la claritas, théorisé par Thomas d'Aquin dans la Somme théologique, se marie dans la conception des vitraux lumineux avec la métaphysique de la lumière d'un 
Pseudo-Denys l'Aréopagite (dont un manuscrit avait été déposé à Saint-Denis au $\mathrm{XI}^{\mathrm{e}}$ siècle et traduit par Jean Duns Scot) ou encore d'un Robert Grosseteste : « De même que la scolastique classique est dominée par le principe de manifestatio, de même l'architecture gothique classique est dominée, comme l'observe déjà Suger, par ce que l'on peut appeler le "principe de transparence" ". Contrairement à l'architecture romane donnant l'impression d'un bastion impénétrable de l'extérieur et uniquement intelligible de l'intérieur, le gothique classique exige que l'espace intérieur « se projette lui-même, en quelque sorte, à travers la structure qui l'enveloppe ; ainsi, par exemple, la coupe transversale de la nef peut se lire sur la façade ${ }^{10} »$. Cette lisibilité du visible s'invertit, dans les écrits spéculatifs, en une visibilité du lisible. Au regard de l'agencement fixe des articles composant la Somme théologique de Thomas d'Aquin, on peut véritablement parler d'une "architecture" totale régissant les traités et s'élaborant par des débuts de paragraphes stéréotypés (le début de l'article par le utrum, l'exposé des objections par le videtur quod non, l'argument contraire par le sed contra, les répliques aux objections par le ad primum, ad secundum etc.). Sans même entrer dans le détail des arguments, la Somme exhibe déjà de loin sa charpente unitaire à l'intérieur de laquelle les arguments résonnent les uns avec les autres. On a souvent relevé l'analogie frappante entre l'évolution graphique et architecturale à l'âge gothique: l'introduction de l'ogive brisée, remontant déjà au début $\mathrm{du} \mathrm{xI}^{\mathrm{e}}$ siècle, correspond à l'apparition des lettrines gothiques qui mettent un terme à la juxtaposition souvent désordonnée des pleins et des vides. Désormais, les manuscrits se présenteront selon une mise en page parfaitement régulière, sans aucun débordement latéral, toute en compacité, mais pouvant se lire également d'un seul coup d'œil en s'orientant le long des grandes lettrines, souvent colorées, qui hiérarchisent les paragraphes.

7 De même que dans la distribution graphique doit se lire la claritas de la pensée, de même la structure externe de la cathédrale doit exhiber son ordre interne. L'ordre cosmique ne connaît aucune interruption et il doit en être pareil de l'organisation du monde sensible par l'homme. Dans l'expression de Suger lux continua, c'est dès lors surtout le "continua» qu'il faut souligner. Les descriptions rassemblées dans les mémoires de l'abbé sont admirablement riches de sens : on peut à la fois les lire comme une apologie de ses activités d'entrepreneur face aux critiques contemporaines (de la part de Saint-Bernard de Clairvaux, pour ne nommer que lui), mais on peut y voir également l'écho de la tradition philosophique néoplatonicienne. Quand il est question de lux nova permettant la continuitas entre le nouveau chœur et la nef, il faut aussi bien y entendre l'amélioration de l'éclairage que la lumière du Nouveau Testament, s'opposant à la cécité de la loi juive, représentée dans l'iconographie par la Synagogue aveugle. On peut alors parler, avec Anca Vasiliu, d'un véritable «idéal de "l'église transparente" » à l'intérieur de laquelle la grâce divine infuse l'espace entier ${ }^{11}$. Les vitraux colorés, associés aux pierres précieuses ornant la Jérusalem Céleste, ne sont autres que le lieu de l'epapogia, d'une ascension par le matériel vers l'immatériel. La nouvelle lumière conduit vers la « vraie lumière [verum lumen] dont le Christ est la vraie porte ».

8 L'appareillage gothique de la transparence est dès lors entièrement orienté vers une transcendance verticale dont la lumière est le reflux. Reprenant littéralement des passages du Pseudo-Denys, les mémoires de Suger témoignent du modèle de l' anagogicus mos, en d'autres termes de l'ascension qui s'effectue toujours, d'après 
l'auteur des Hiérarchies Célestes, « du matériel vers l'immatériel ». Sept siècles plus tard, les versions profanes de ces temples de la lumière sont construites à travers l'Europe. Ici, point de tension verticale : l'utopie est toute entière déployée à l'horizontale.

\section{L'Eden sous cloche}

«La serre, avec sa végétation, a été en tant que symbole du jardin d'Eden le point de convergence de toutes les utopies sociales du XIX siècle »

Georg Kohlmaier

Toute révolution a ses antécédents. Lorsque Vladimir Ilitch Lénine écrit son Que faire?, il emprunte le titre à l'ouvrage homonyme publié en 1863 par Nikolaï Tchernychevski ${ }^{12}$. Dans ce roman allégorique qui allait devenir le livre de chevet de tous les révolutionnaires (Lénine assure en avoir été "labouré de fond en comble ${ }^{13}$ ), Tchernychevsky dépeint les visions utopiques de son héroïne Vera Pavlovna. Ainsi découvre-t-on dans le Quatrième Songe la description d'un Palais de Cristal à l'intérieur duquel se déploie une société heureuse et laborieuse, secondée par des machines qui transforment les déserts en oasis et les montagnes escarpées en prairies fleuries sur lesquels gambadent gaiement hommes et femmes en tuniques à l'antique tandis que l'air résonne de chants radieux. Point de miasmes, point de maladies dans ce monde pur et limpide : le couronnement du dôme de cristal est constitué par un large panneau dépoli, aménagé pour diffuser la lumière dans l'espace entier.

Crystal Palace

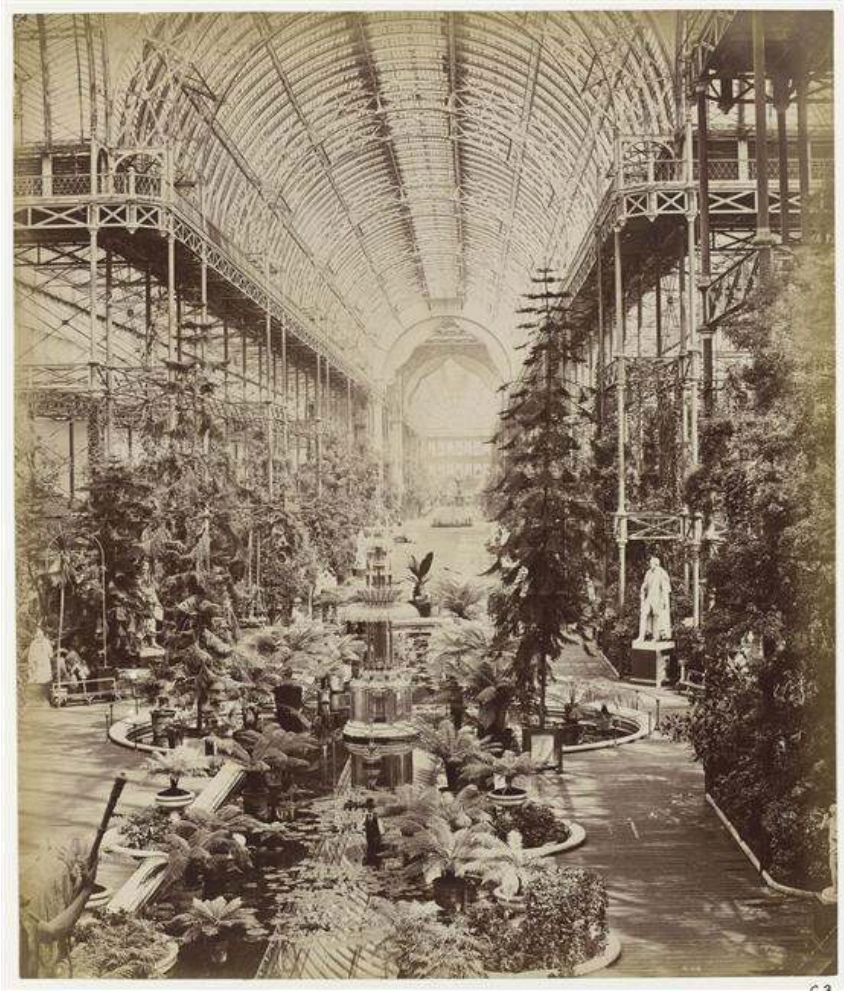

Hervé Lewandowski, Crystal Palace, Londres, intérieur, 1851

10 Ce dôme de cristal n'est cependant pas né entièrement de l'imagination de son auteur. En 1854, Tchernychevski avait rédigé pour les lecteurs des Annales de la Patrie un 
compte-rendu de l'exposition industrielle de Londres en $1851^{14}$. Depuis son inauguration, les gazettes du Vieux et du Nouveau Monde ne tarissent plus en évocations superlatives de ce qui constitue la sensation de l'exposition : le Crystal Palace, cette serre entièrement réalisée en verre et en fer qui devait être, selon le désir du prince Albert et de Henry Cole, instigateurs de l'exposition, le bâtiment le plus vaste de l'univers. À neuf mois de l'inauguration, tous les 245 projets de palais sont refusés. Le but fixé par les idéateurs de l'exposition semble inatteignable, lorsque que ceux-ci décident, dans un dernier élan, de faire appel à Joseph Paxton. La reine Victoria avait en effet pu admirer le nouveau type de serre que ce jardinier du Devonshire avait mis au point quelques années auparavant et qui reposait sur un système de structures, elles-mêmes préfabriquées par une machine inventée par Paxton. La reine était restée stupéfaite par cette serre de 85 mètres de long sur 38 de large et 20 de haut, dimensions jusque-là inédites. Bien que considérables par rapport aux serres habituelles, la construction de Paxton était toutefois loin des conditions requises pour l'exposition (563 mètres de long sur 124 mètres de large !). En sept jours et sept nuits, Paxton réalise alors ce qui ressemble à une immense boîte de construction avec 3.300 piliers de fer, 2.224 poutrelles, 205.000 cadres de bois avec autant de carreaux de verre. On parle du premier préfabriqué industriel de l'histoire.

Dans la Revue des deux mondes de 1851, on peut lire les impressions exaltées d'un des centaines de milliers de visiteurs qui découvriront le palais de verre : «À l'extérieur, figurez-vous un jardin d'hiver grand comme le jardin des Tuileries... Voici des arbres d'Europe, énormes et touffus [Paxton avait construit ses serres par-dessus les ormes centenaires qui s'y dressaient déjà] qui étendent en toute liberté leur feuillage sous ces voûtes transparentes ». Mais cette serre ne protège pas uniquement des espèces botaniques, elle est le présentoir de toutes les marchandises venant des colonies de l'Empire: "Imaginez des lieues entières de tapis de toute sortes, de cristaux resplendissants, de meubles d'une richesse insensée, de bronzes, de velours, de porcelaines, de soieries, de tissus d'argent et de perles, des bijoux dignes de Cléopâtre, de diamants de Golconde ${ }^{15}$ ». Lothar Bucher, un exilé allemand qui allait devenir plus tard le bras droit de Bismarck au ministère des Affaires étrangères, assista à l'inauguration le $1^{\mathrm{er}}$ mai 1851 et résume la sensation à laquelle est exposée le visiteur : « Les murs latéraux sont trop éloignés pour qu'on puisse les embrasser d'un seul regard et au lieu de buter sur le mur opposé, l'œil glisse le long d'une perspective infinie qui se perd dans la brume ${ }^{16}$ ». Et de poursuivre plus loin: «Si nous le laissons s'abaisser progressivement, notre regard rencontre les poutrelles ajourées, peintes en bleu, séparées d'abord par de larges intervalles, se rapprochant ensuite jusqu'à se superposer, puis soudain interrompues par une brillante bande de lumière - la nef transversale - pour finir par se fondre dans un lointain où tout devient immatériel ${ }^{17}$ ". On croit lire du Suger de Saint-Denis évoquant sa nouvelle basilique.

La différence est néanmoins de taille : là où la cathédrale gothique est érigée comme monument résistant au temps séculier, la nouvelle architecture de verre et de fer Walter Benjamin le souligne - est pensée en fonction du transitoire : « Pour la première fois depuis les Romains un nouveau matériau de construction artificiel, le fer, fait son apparition" fait-il remarquer dans Paris, capitale $d u X x^{e}$ siècle $^{18}$. On l'évite pour la construction des lieux d'habitation traditionnels (le fer et le verre semblent marqués à tout jamais par le seing de l'extériorité, de la froideur) pour l'utiliser plutôt « pour les passages, les halls d'exposition, les gares - toutes constructions qui visent à des buts 
transitoires ${ }^{19}$ ». Benjamin ne manque pas de voir que le palais de Paxton en constitue l'archétype : à la fois hall, passage et cathédrale profane, il ne s'arc-boute au-dessus d'aucun lieu privilégié mais a pour fonction de mettre en valeur ce qu'il recouvre en se faisant oublier. Cette paroi mallarméenne, fruit de la production industrielle de masse, peut être démontée en pièces détachées et recomposée à souhait. Après l'Exposition, le Crystal Palace fut ainsi reconstitué à Sydenham, en 1854, d'après des plans modifiés par Paxton qui ajouta neuf transversales supplémentaires. Il y restera jusqu'en 1936, lorsqu'un incendie le détruira.

La haute société londonienne se dispute par articles interposés sur le meilleur usage de ces $700.000 \mathrm{~m}^{2}$ que recouvrira le Palais de Cristal reconstitué. Son inventeur n'est pas en reste : dans son What is to become of the Crystal Palace?, Joseph Paxton déploie sa vision idéale: « Nous y créerons le climat de l'Italie du Sud où les masses pourront monter à cheval, se promener ou encore se reposer sous les feuillages d'arbres exotiques et y contempler tranquillement les œuvres de la nature et de l'art, sans être tourmenté par les vents d'ouest ou des bourrasques de neige ${ }^{20} »$. Les plans de Paxton sont retenus; contrôlé par une société d'actionnaires, Sydenham deviendra le premier parc de loisirs et de récréation de masse, avec ses oiseaux exotiques, ses expositions géographiques et ses collections ethnologiques, mais également la présentation des derniers produits industriels. De la sorte, ce qui n'était qu'un éphémère pavillon d'exposition se trouve pérennisé tandis que le passage se voit institutionnalisé comme espace unique de libre circulation entre les produits de consommation. L'alliage entre l'opacité du fer et la légèreté du verre, écrivait Marx dans une de ses revues pour la Neue Rheinische Zeitung, permet l'érection d'un temple érigé à la gloire de la marchandise, un «Panthéon dans la Rome moderne » où la bourgeoisie mondiale expose « avec un fier contentement, les dieux qu'elle s'est fabriqués pour elle-même ${ }^{21} »$, c'est-à-dire le caractère fétiche (dont l'étymologie, pour rappel, dérive du mot portugais signifiant «fabriqué ») de la marchandise.

Le lien étroit entre le caractère fantasmatique et la présentation immaculée qu'offre le verre a été fréquemment souligné par Walter Benjamin. Dans les vitrines des rues parisiennes, le flâneur découvre les objets de son désir et son image reflétée dans la vitre finit par se superposer à l'image de l'objet désiré. Dans le palais de Paxton, le verre qui constituait encore la paroi séparant le passant de son objet disparaît pour devenir la membrane qui, en les enveloppant, les met dans une situation de rapport immédiat. La marchandise n'est plus intouchable parce qu'elle se trouve derrière mais bien parce qu'elle se tient sous le verre. L'interdiction de toucher, la prohibition d' utiliser les objets exposés ne dépend plus d'une séparation matérielle mais relève d'une impossibilité littéralement " sublimée ", relevée à la voûte de verre dont elle est la clef. Benjamin précise: "Les expositions universelles idéalisent la valeur d'échange des marchandises. Elles créent un cadre où leur valeur d'usage passe au second plan. Les expositions universelles furent une école où les foules écartées de force de la consommation se pénètrent de la valeur d'échange des marchandises jusqu'au point de s'identifier avec elle : 'il est défendu de toucher aux objets exposés'. Elles donnent ainsi accès à une fantasmagorie où l'homme pénètre pour se laisser distraire ${ }^{22}$ ». Sous le dôme de cristal, point de production ni de consommation mais un état homéostatique de réciprocité entre le prolétarien, paria et intouchable, et les biens exposés, intouchables à leur tour. Tout en maintenant toutes les différences de statut à l'extérieur de l'architecture de verre, celle-ci agit comme un égalisateur social en son sein où les règles sont égales pour tous, ce qui explique que les saint-simoniens de la 
revue Le Globe aient massivement soutenu la visite des expositions universelles et la construction de parcs sur le modèle de Sydenham. Le verre aura donc désormais moins pour but de laisser pénétrer la lumière de l'extérieur que d'en atténuer l'effet individuant et de ménager un espace de pure égalité. "À l'intérieur des divertissements, auxquels l'individu s'abandonne dans le cadre de l'industrie de plaisance, il reste constamment un élément composant d'une masse compacte» et s'il ne peut posséder ce qui est sous verre, l'individu perdu dans la masse oublie un instant qu'il est avant tout une force productrice pour s'abandonner à la passivité de la distraction: «Cette masse se complaît dans les parcs d'attraction avec leurs montagnes russes, leurs 'tête-à-queue', leurs 'chenilles', dans une attitude dénuée de toute réaction. Elle s'entraîne par là à cet assujettissement avec lequel la propagande tant industrielle que politique doit pouvoir compter ${ }^{23} »$. D'où une troublante proximité entre la dimension égalisatrice et utopique et son élément cynique, préfigurant les centres de loisir du $\mathrm{xx}^{\mathrm{e}}$ siècle tardif.

Incendie du Crystal Palace

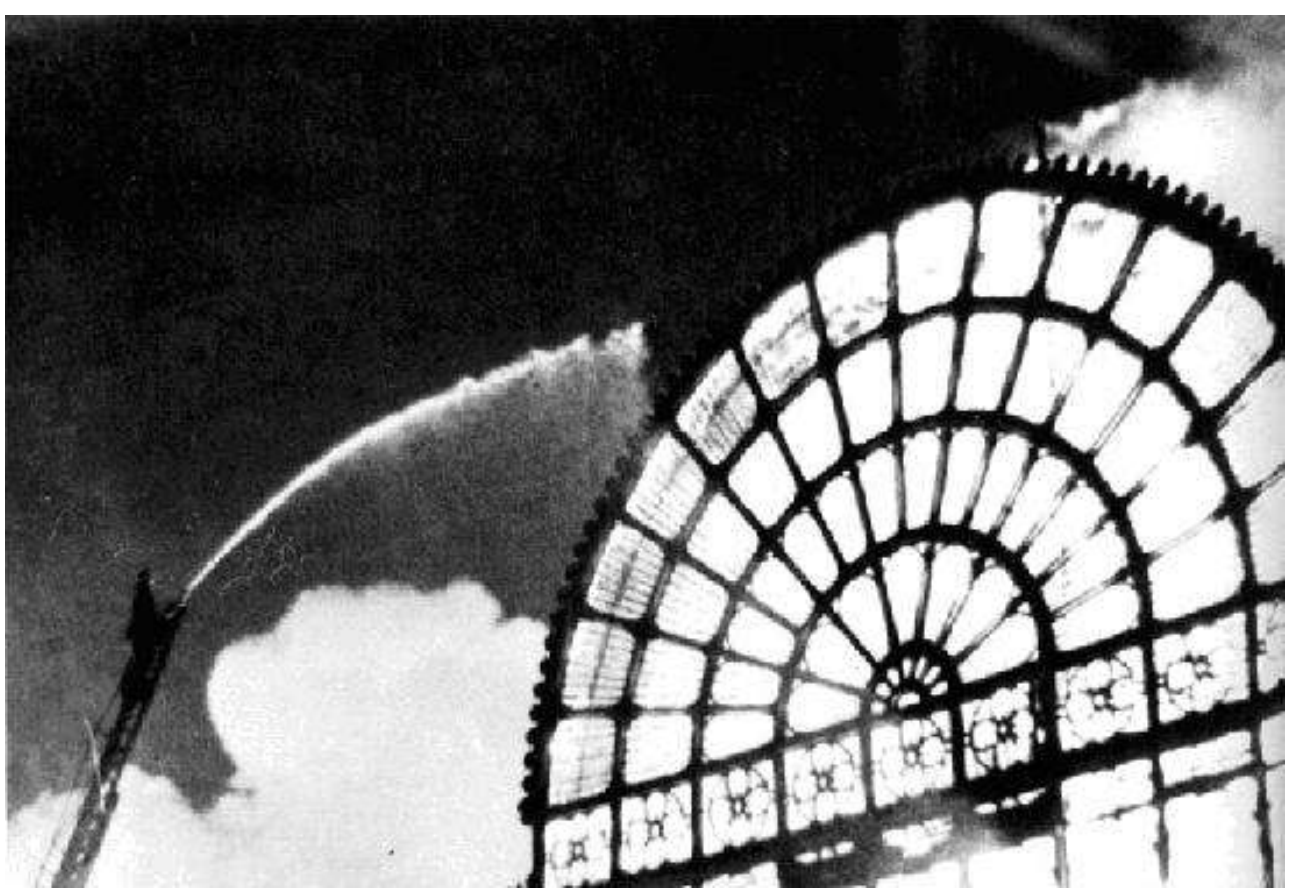

Anonyme, Incendie du Crystal Palace (détail), tiré du Illustrated London News du 5 décembre 1936

Dostoïevski, qui passera brièvement par Londres en 1862 lors de l'exposition universelle et visita selon toute vraisemblance le Crystal Palace à Sydenham, traduit cette ambivalence dans ses incisives Notes d'hiver sur des impressions d'été:

Vous reconnaissez une pensée gigantesque ; vous sentez qu'ici quelque chose a déjà été obtenu, qu'ici il y a la victoire, le triomphe. Vous paraissez même commencer à craindre quelque chose. [...] Ceci ne serait-il pas l'idéal atteint ? Pensez-vous ; n'estce pas la fin? Ne serait-ce pas l'« unique troupeau»? Ne faudrait-il pas l'accepter en effet comme vérité parfaite et se taire définitivement? Tout cela est si triomphant, si victorieux et si fier, que vous commencez à respirer avec peine. Vous regardez ces centaines de mille, ces millions d'hommes, qui y viennent humblement de toute la surface terrestre - des hommes venus avec une seule pensée, qui se tiennent silencieux et avec un calme entêtement, dans ce palais colossal, et vous sentez, que quelque chose de définitif s'est accompli, accompli et terminé. [...] Vous 
sentez qu'il faudrait énormément de résistance et de négation séculaires, pour ne pas se soumettre, ne pas céder à l'impression, ne pas s'incliner devant le fait et ne pas adorer Baal, c'est-à-dire ne pas prendre ce qui existe pour son idéal ${ }^{24}$. une alimentation secrète de son écriture ${ }^{25}$, tout particulièrement dans les Carnets $d u$ sous-sol qui se présentent comme distorsion grinçante de l'utopie de la société transparente dépeinte par Tchernychevski (qui ne manque de rappeler, dans le Sixième rêve de Vera Pavlovna, qu'il y a à Sydenham un palais de cristal ressemblant très fortement à celui rêvé par la jeune révolutionnaire). Face à l'héroöne de Que faire?, l'« anti-héros » dostoïevskien habite une cave aveugle, revers saturnien du temple de verre. Non moins lucide pour autant, l'auteur des Carnets dénonce l'hyperrationalisation masquée dans cette utopie du progrès enfermant l'homme dans un étau fonctionnel pour le réduire à n'être plus qu'une "touche de piano", une « vergette d'orgue » intégrée à un vaste déterminisme machinal.

\section{La civilisation de verre}

«Vivre dans une maison de verre est, par excellence, une vertu révolutionnaire. » Walter Benjamin

À l'orée $\mathrm{du} \mathrm{xx}^{\mathrm{e}}$ siècle, d'autres écrivains émirent toutefois un jugement moins intransigeant sur le verre que l'auteur de Crime et châtiment et le principe de la transparence devient même le signe d'une certaine littérature voulant se démarquer de force des vestiges de l'intériorité qui collaient encore aux décadentistes autour de KarlJoris Huysmans. Dès les premières pages de Nadja, André Breton se lance dans l'éloge du verre, panacée de la nouvelle société : « Pour moi, je continuerai à habiter ma maison de verre, où l'on peut voir à toute heure qui vient me rendre visite, où tout ce qui est suspendu aux plafonds et aux murs tient comme par enchantement, où je repose la nuit sur un lit de verre aux draps de verre ${ }^{26} »$. Dans son article sur Breton et le surréalisme, Walter Benjamin notait « Vivre dans une maison de verre est, par excellence, une vertu révolutionnaire. Cela aussi est une ivresse, un exhibitionnisme moral dont nous avons grand besoin. La discrétion sur ses affaires privées, jadis vertu aristocratique, est devenue de plus en plus le fait de petits-bourgeois arrivés ${ }^{27} »$. Mais peut-être plus encore que dans Nadja de Breton, c'est dans les poèmes du futurisme italien que Benjamin aurait trouvé la confirmation de son intuition. Quinze ans avant le roman de Breton, Aldo Palazzeschi, aède militant du futurisme italien, avait composé un poème, La petite maison de cristal, qui présente le verre comme l'antidote contre toute retombée dans l'intérieur calfeutré du romantisme :

Je rêve d'une maison de cristal [...] Une maison comme tout commun mortel/ pourrait posséder,/ qui n'aurait rien d'extraordinaire,/ mais qui serait toute transparente,/ en cristal./ [...] Vous me verrez manger, vous pourrez me voir/ quand je dors,/ surprendre mes rêves;/ vous me verrez en train de faire mes besoins,/ vous me verrez en train de changer de chemise ${ }^{28}$.

Le verre qui instituait encore, dans le palais de Paxton, un espace d'exception, devient dans ces visions d'utopies sociétaires le matériau même des architectures quotidiennes et se voit étendu à tous les domaines.

$\mathrm{Au}$ même moment où les futuristes élaborent les prémisses d'une nouvelle alliance entre la technique, les arts et l'organisation sociale, un groupe se constitue à Berlin autour de Paul Scheerbart et Bruno Taut intitulé Die gläserne Kette («La chaîne de 
Verre ») visant à jeter les bases d'une nouvelle civilisation du verre. En 1914, Scheerbart avait publié son manifeste Glasarchitektur («Architecture de verre»), mélange déconcertant de méditation historico-philosophique, de programme industriel mégalomaniaque et de fantaisie littéraire où l'impulsion générale est toutefois expliquée d'entrée de jeu avec beaucoup de sobriété :

Nous vivons le plus souvent dans des espaces clos, qui constituent le milieu où s'enracine et se développe notre civilisation. Notre civilisation est dans une certaine mesure un produit de notre architecture; si nous voulons élever son niveau, nous devons donc, bon gré mal gré, transformer notre architecture. Et cela ne sera possible que si nous faisons en sorte que les pièces dans lesquelles nous vivons n'aient plus ce caractère $\operatorname{clos}^{29}$.

Les fenêtres (séparation entre intérieur et extérieur) seront abolies, les portes seront en verre transparent et des aéronefs flotteront au-dessus des villes équipés de projecteurs de couleur, plongeant les hommes dans une atmosphère chatoyante et un « milieu » coloré en permanence.

21 La même année, Bruno Taut présente à Cologne sa Maison de Verre à l'exposition du Werkstattbund. Les commentateurs y voient comme la réalisation du songe de Scheerbart. Dans la forme circulaire du toit à forme d'asperge se traduit le bagage expressionniste et spiritualiste, tandis que les projets de «désurbanisation » de Taut font plutôt signe vers les doctrines anarchistes de Kropotkine auquel l'architecte se réfère par ailleurs. Les deux se rejoignent dans le rêve d'une "communauté organique » opposé à la société triomphante des civilisations industrielles, opposition qui remonte à l'ouvrage influent de Ferdinand Tönnies Gemeinschaft und Gesellschaft (Communauté et société) $^{30}$.

\section{Esquisse pour un gratte-ciel, Friedrichstrasse}

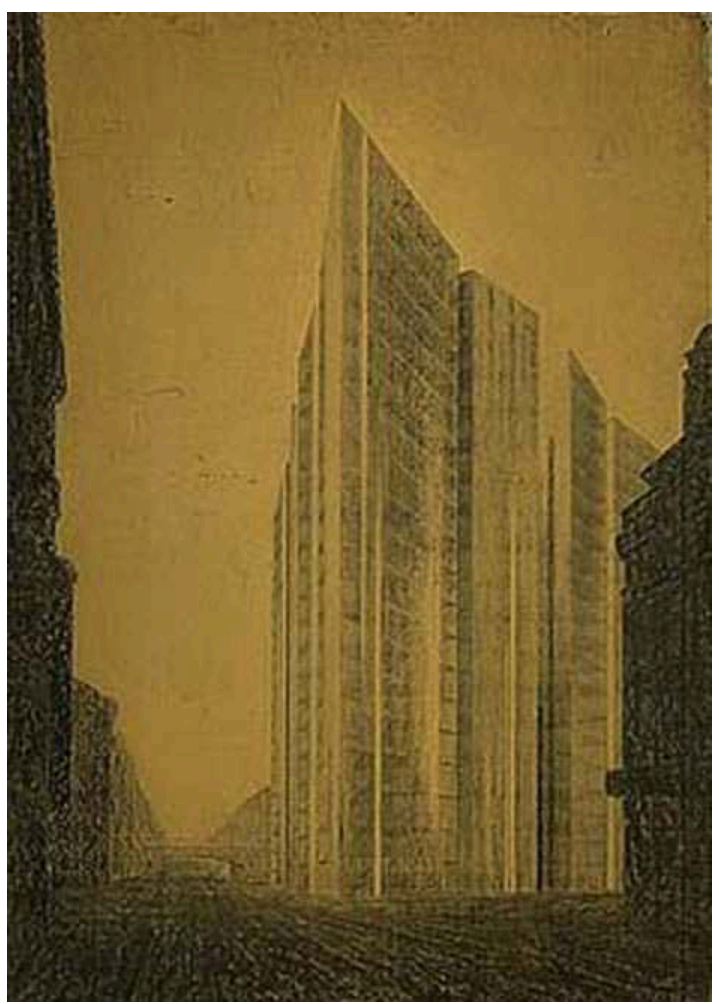

Mies van der Rohe, Esquisse pour un gratte-ciel, Friedrichstrasse, Berlin, 1921. 
En utilisant les mêmes nouvelles possibilités techniques, Mies van der Rohe propose en 1921 un plan jamais réalisé d'un gratte-ciel entièrement revêtu de verre pour la Friedrichstrasse à Berlin. Loin des dérives spiritualistes d'un Bruno Taut, Mies veut au contraire une architecture anonyme, abstraite. Dans différentes revues (dont le « Frühlicht » publié par Taut), les principes de ce nouvel art de bâtir sont énoncés :

Le bureau est une maison de travail, d'organisation, de clarté, d'économie. Des espaces de travail vastes et clairs, intelligibles, indivis et uniquement organisés comme l'organisme de l'entreprise. Le plus grand effet avec le minimum de moyens. Les matériaux sont le ciment, le fer, le verre ${ }^{31}$.

Si, de prime abord, les principes rationnels de Mies semblent être loin des rêveries d'un Scheerbart, Benjamin lui avait bien vu, dans cet extraordinaire petit texte qu'est Expérience et pauvreté, ce qui reliait l'utopiste fantasque à la sobriété des Mies, Loos et autres Corbusier. «Scheerbart avec son verre, le Bauhaus avec son fer, sont venus à bout [de l'intérieur bourgeois], ils ont créé des espaces où il est difficile de laisser des traces $^{32} »$. On retrouve ici la théorie de l'intérieur et de la trace, déjà brossée quelques années auparavant dans un fragment de Brèves ombres: "Lorsqu'on pénètre dans le salon bourgeois des années 1880 , quelle que soit l'atmosphère de douillette intimité qui s'en dégage, l'impression dominante est : 'Tu n'as rien a faire ici'. Tu n'as rien à y faire, parce qu'il n'est pas de recoin où l'habitant n'ait déjà laissé sa trace ${ }^{33}$ ». La devise de l'homme moderne est donnée par Brecht dans son Manuel pour les habitants des villes: «Efface tes traces!». Il n'est donc pas fortuit, poursuit Benjamin, si Loos et Le Corbusier ont réalisé les «maisons de verre mobiles » imaginées par Scheerbart : «Le verre, ce n'est pas un hasard, est un matériau dur et lisse sur lequel rien n'a prise. Un matériau froid et sombre également ». "Le verre d'une manière générale » poursuit-il «est l'ennemi du mystère ${ }^{34}$ ». Et d'intercaler une phrase, qui paraît presque anodine, mais dont on mesure toute la portée pour l'auteur de L'Euvre d'art à l'époque de la reproductibilité technique : «Les objets de verre n'ont pas d"aura' ${ }^{35}$ ".

Benjamin n'a cessé d'avoir un rapport ambigu au verre sous toutes ses formes ${ }^{36}$. Toutefois, sa foi dans sa dimension utopique, inséparable de son côté aliénant, est restée inébranlée, car le verre - écrit-il encore dans l'essai où il théorise la "bonne " pauvreté - « est aussi l'ennemi de la propriété. Le grand écrivain André Gide a dit un jour : chaque objet que je veux posséder devient opaque. Si des gens comme Scheerbart rêvent de constructions en verre, serait-ce parce qu'ils sont les apôtres d'une nouvelle pauvreté $?^{37} »$. Ce qui est sous verre ne se laisse posséder et Scheerbart préfigurerait ainsi l'abolition de la propriété privée: dans la notice sur Scheerbart, celui-ci se voit même comparé à la société libre de Fourier ${ }^{38}$.

Benjamin aurait sans doute eu plus de réticences pour en dire autant des architectures de verre des années 1960 .

\section{Transparency international}

« Glasnost ! " Michaël Gorbatchev

L'essor de l'industrie automobile et l'accroissement exponentiel de la vitesse des véhicules avait propulsé dans les années 30 les recherches sur le vitrage sécurisé. Les verreries de Saint-Gobain travaillent dès 1929 sur la trempe de verre, mais c'est finalement le brevet déposé par Alastair Pilkington en 1955 pour la fabrication du verre 
flotté qui sonnera définitivement le glas des vitres cassées d'antan. Les audacieux projets de gratte-ciel en verre de Mies van der Rohe, restés à l'état d'ébauche avant la guerre, sont désormais à portée de main. Mies lui-même bâtit à New York le Seagram Building (1958); la transparence qui lui était si chère dans les années 20 semble être toutefois passée au second plan et c'est au contraire l'effet de structure, obtenu par une charpente intérieure en bronze, qui est souligné. À quelques centaines de mètres de là se dresse un autre gratte-ciel qui prolonge les visions d'une architecture transparente : le Lever Building du bureau Skidmore, Owings et Merrill dont la surface est constituée d'un filet filigrane totalement détaché de l'ossature. Ces vitres qui suggèrent la pénétration totale par le regard n'en sont pas moins infrangibles. Richard Sennett en a fait, dans son essai Architectures de verre, le symbole de la protection de l'individu contre l'attrait du dehors: "Le gratte-ciel devrait fatalement attirer les suicides; les tours s'élancent haut dans le néant, leur hauteur même créant un abîme au-dessous. Mais le verre plan [plate glass, verre "sécurit »] constitue la grande ressource moderne contre le suicide et de moindres actes de spontanéité. Il n'est pas facile de pulvériser une fenêtre de verre plan; quant à détacher le verre de son encadrement métallique, cela demande de la patience et du métier. Le candidat au suicide, qui en aurait soudain assez de l'existence, aura beau presser le front désespérément contre le verre, il n'en demeure pas moins protégé par un matériau qui lui fait voir, toute en le rendant accessible, l'objet de son désiri ${ }^{39}$ ". Là où la vitrine des passages protégeait au XIX ${ }^{\mathrm{e}}$ siècle l'objet désiré de l'individu, c'est à présent l'individu qui est préservé de son désir par la vitre sécurisée. L'artiste américain Dan Graham - dont un pan d'œuvre important est dédié à la réflexion sur le verre et ses architectures - a mis en relief l'absence d'extériorité qui se cache sous cette apparente mise en relation de l'intérieur et de l'extérieur par le principe de transparence. Anticipant l'essoufflement des brandings, logos et autres images de marque, Graham s'était intéressé, dans la foulée d'un travail vidéo, à ces nouveaux signes de reconnaissance corporatifs que sont les architectures de verre. Loin de renoncer à une image de marque propre, ces entreprises optant pour le principe de la transparence font confiance au pouvoir métonymique de la surface transparente. Rien n'est caché au client, pas de publicité mensongère, les biens de consommation sont sous ses yeux et ne lui restent plus extérieurs. "L'immeuble de verre, indépendant et transparent, refuse d'admettre une apparence extérieure en tant qu'élément constitutif » - écrit Graham dans Immeubles de verre : 'vitrines' d'entreprises "quand d'autres immeubles sont généralement décorés de signes conventionnels indiquant leur fonction afin que le public se repère, la façade de l'immeuble de verre est virtuellement éliminée ${ }^{40} \%$. L'apparence de l'immeuble, sa propreté et sa transparence structurelle allient donc le mythe de l'avancée technologique à celui de l'« efficacité ». En tant qu'instance de surveillance, le passant est, semble-t-il, incorporé dans le rouage de l'entreprise et en devient une partie intégrante.

Une nouvelle étape dans l'histoire de la surveillance, ébauchée magistralement par Foucault dans son Surveiller et punir, reste à écrire. Dans ses plans pour un pénitencier idéal, le Panoptique, Jeremy Bentham avait procédé à une orientation centripète de toutes les cellules d'internement vers une tour centrale d'où un gardien unique pouvait désormais contrôler de son regard tous les détenus. Dans un tel dispositif centralisateur, l'appareil de surveillance humain est réduit à l'unité numéraire pour tendre à sa disparition même : l'impénétrabilité de la tour rejette l'incarcéré sur luimême et le place, dans son impossibilité de pouvoir vérifier si un gardien est réellement présent, dans une situation de surveillance virtuelle permanente. Face à 
l'organisation focale des alvéoles pénitentiaires, les instances de contrôle sont doublement décentrées dans les immeubles transparents. Un décentrement interne d'abord, les bureaux ayant été décloisonnés (la célèbre paroi derrière laquelle se réfugiait Bartleby pour se séparer des autres employés de l'office notarial disparaissant définitivement) et les subordonnés étant exposés désormais non seulement au regard d'un superviseur mais de tous les autres travaillant dans le même espace unique et traversant. Un décentrement extérieur ensuite, cet espace n'étant séparé du dehors que par une paroi de verre tandis que chaque employé est dorénavant soumis à l'œil du passant dont le regard s'arrêterait par hasard sur son activité.

À l'endroit où Walter Benjamin se tenait dans les années ' 20 pour décrire le va-et-vient incessant des trams électriques au Potsdamer Platz à Berlin, se dresse aujourd'hui la peau immaculée du Sony Center en verre et en acier. C'est à présent le flâneur, si magnifiquement théorisé par Benjamin, qui joue, lorsqu'il passe devant ces offices translucides, le rôle d'un superviseur, fuyant, mais panoptique encore, des nouvelles réalités du travail. Or en vérité, point de réciprocité visuelle dans le dispositif de la transparence. Cette dernière est affichée et dévisagée, mais ceux sont à l'intérieur n'ont pas pour fonction de voir, mais uniquement d'être vus, le théâtre réel des prises de décisions étant ailleurs, en dehors du champ visuel, ce qu'avait très bien remarqué Dan Graham :

La transparence absolue est seulement visuelle ; le verre sépare le visuel du verbal, et isole les étrangers du contenu des processus de prise de décision et des interrelations invisibles, mais réelles qui relient les opérations commerciales et la sociétét ${ }^{41}$.

\section{Potsdamer Platz}

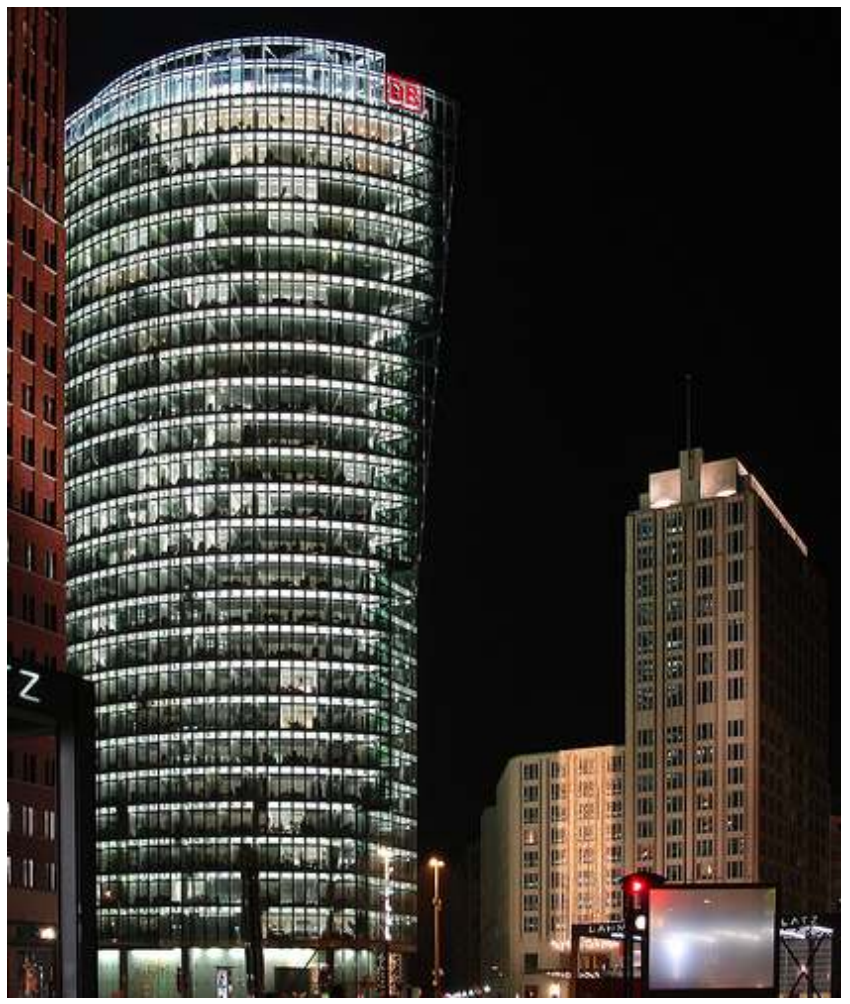

Gertrud Kanu, Potsdamer Platz, Berlin, 2006

(C) Courtesy of Gertrud Kanu 
batiment des Nations Unies à New York soit alors passé de la match box aux deux côtés aveugles à la glass box aux quatre faces de verre n'y changera guère beaucoup mais confirmera plutôt le constat que le discours sur la transparence - valeur suprême et inattaquable de notre époque - a pour essentielle et principale fonction de dissimuler le maintien des rapports de forces, nouvelles dans leurs formes, mais identiques dans leur inégalité.

Note : Cet article paraît simultanément en anglais sous le titre "Architectures of transparency » dans la revue RES. Aesthetics and Anthropology 53 (2008)

\section{BIBLIOGRAPHIE}

Benjamin Walter, « Brèves ombres », M. de Gandillac (trad.), revue par P. Rusch, in Euvres II, Paris, Gallimard, 2000, p. 349-354.

Benjamin Walter, « Expérience et pauvreté », P. Rusch (trad.), in CEuvres II, Paris, Gallimard, 2000, p. 354-372.

Benjamin Walter, « Paul Valéry. Pour son soixantième anniversaire », M. de Gandillac (trad.), revue par P. Rusch, dans Euvres II, Paris, Gallimard, 2000, p. 322-329, p. 328.

Benjamin Walter, «Sur Scheerbart », in Écrits français, Paris, Gallimard, 1991, p. 324-327.

Benjamin Walter, "Paris, capitale du XIX ${ }^{\mathrm{e}}$ siècle », in Gesammelte Schriften, R. Tiedemann (ed.), vol. V, 1, Francfort, Suhrkamp, 1982 (réédition en format poche : Paris capitale du XIXe siècle, Paris, Allia, 2003).

Benjamin Walter, « Le Surréalisme. Le dernier instant de l'intelligentsia européenne », M. de Gandillac (trad.), revue par P. Rusch, in Euvres II, Paris, Gallimard, 2000, p. 113-134.

Bloch Ernst, Le principe espérance. II : Les épures d'un monde meilleur, F. Wuilmart (trad.), Paris, Gallimard, 1982.

Breton André, Nadja, Paris, Gallimard, 1963.

Catteau Jacques, « Du palais de cristal à l'âge d'or ou les avatars de l'utopie », in Dostoïevski, J. Catteau (dir.), Paris, L'Herne, 1973, p. 176-195.

Dan Graham, «Glass Buildings: Corporate "Showcases"/Immeubles de verre : "vitrines" d'entreprises ", in Ma position. Écrits sur mes œuvres, Villeurbanne, Nouveau Musée/Les presses du réel, 1992, p. 144-147.

De Bruyne Edgar, Études d'esthétique médiévale. III: Le XIII siècle, Genève, Slatkine, 1975 (réimpression de l'édition de 1946).

Déotte Jean-Louis, L'Homme de verre. Esthétiques benjaminiennes, Paris, L'Harmattan, 1998. Dostoïevsky Fiodor, Notes d'hiver sur des impressions d'été, W. Bienenstock (trad.), notes par C. Meyer, Paris, L'Entente, 1988.

Giedion Siegfried, Espace Temps Architecture, I. Lebeer et F.-M. Rosset (trad.), Paris, 1978. 
Grodecki Louis, Architecture gothique, avec la collaboration d'A. Prache et de R. Recht, Paris, Gallimard/Milan, Electa, 1992.

Jantzen Hans, Über den gotischen Kirchenraum und andere Aufsätze [1927], Berlin, 1951.

Kohlmaier Georg, von Sartory Barna, Das Glashaus. Ein Bautypus des 19. Jahrhunderts, Munich, Prestel, 1981.

Marx Karl, Neue Rheinische Zeitung, Politisch-ökonomische Revue, $\mathrm{n}^{\text {os }}$ 5-6, mai-octobre 1850, in Cuvres IV. Politique I, M. Rubel (ed), Paris, Gallimard, 1994.

Mies van der Rohe, Das kunstlose Wort. Gedanken zur Baukunst, F. Neumeyer (ed.), Munich, Siedler, 1986.

Missac Pierre, «L'architecture de verre », in Passage de Walter Benjamin, Paris, Seuil, 1987, p. 157-184.

Palazzeschi Aldo, « Una casina di cristallo (congedo) », in Tutte le poesie, A. Dei (éd.), Milan, Mondatori, 2002, p. 316-319.

Panofsky Erwin, Architecture gothique et pensée scolastique, P. Bourdieu (trad. et postface), Paris, Minuit, 1967.

Scheerbart Paul, L'architecture de verre, P. Galissaire (trad.), Strasbourg, Circé, 1995.

Sedlmayr Hans, Die Entstehung der Kathedrale, Zurich, Atlantis, 1950.

Sennett Richard, « Architectures de verre », in Le temps de la réflexion, VIII, Paris, Gallimard. 1983, p. 125-140.

Simson Otto von, The gothic cathedral. Origins of gothic architecture and the mediaeval concept of order, New York, 1956.

Tafuri Manfredo, Projet et utopie. De l'Avant-garde à la Métropole, F. Brun (trad.), Paris, Bordas, 1979.

Tchernychevski Nikolaï, Que faire? Les hommes nouveaux (1863), D. Sesemann (trad), Paris, Syrtes, 2000.

Vasiliu Anca, « Le mot et le verre. Une définition médiévale du diaphane », in Journal des savants 1994/1, p. 135-162.

Vidler Anthony, « Transparency », in The Architectural Uncanny: Essays in the Modern Unhomely, Cambridge/Mass., MIT Press, 1992, p. 217-226.

\section{NOTES}

1. Benjamin Walter, «Paul Valéry. Pour son soixantième anniversaire » [1931], M. de Gandillac (trad.) revue par P. Rusch, dans Euvres II, Paris, Gallimard, 2000, p. 322-329, p. 328.

2. Vidler Anthony, "Transparency », in The Architectural Uncanny: Essays in the Modern Unhomely. Cambridge/Mass., MIT Press, 1992, p. 217-226.

3. Ibid., p. 168.

4. Ibid., p. 217.

5. Panofsky Erwin, Architecture gothique et pensée scolastique, P. Bourdieu (trad. et postface), Paris, Minuit, 1967, p. 50

6. «Pars noua posterior dum iungitur anteriori, aula micat mediu clarificata suo. Claret enim claris quod clare concopulantur/ Et quod perfundit lux noua, claret opus/ Nobile [...]/ » De administratione 181 . 
7. «illo urbano et approbato in circuitu oratoriorum incremento, quo tota clarissimarum uitrearum luce mirabili et continua interiorem perlustrante plucritudinem eniteret » (Suger De consecratione 49).

8. Les deux textes respectivement de 1946 et de 1948 ont été réunis dans Panofsky Erwin, Architecture gothique et pensée scolastique. D'autres auteurs ont défendu des thèses analogues: Jantzen, Hans: Über den gotischen Kirchenraum und andere Aufsätze [1927], Berlin, 1951. Sedlmayr Hans, Die Entstehung der Kathedrale, Zurich, Atlantis, 1950. Simson Otto von, The gothic cathedral. Origins of gothic architecture and the mediaeval concept of order, New York, 1956. De Bruyne Edgar, Études d'esthétique médiévale. III: Le XIII siècle, Genèv, Slatkine, 1975 (réimpression de l'édition de 1946), p. 3-29. Pour une approche plus critique, fondée sur des recherches historiques, voir Grodecki Louis, Architecture gothique, avec la collaboration d'A. Prache et de R. Recht, Paris, Gallimard/Milan, Electa, 1992.

9. Panofsky, Architecture gothique et pensée scolastique, p. 102.

10. Ibid., p. 103.

11. Vasiliu Anca, "Le mot et le verre. Une définition médiévale du diaphane ", in Journal des savants 1994/1, p. 135-162, p. 154.

12. Tchernychevski Nikolaï, Que faire? Les hommes nouveaux (1863), D. Sesemann (trad.), Paris, Syrtes, 2000.

13. Ibid., p. 11 (préface de Yolène Dilas-Rocherieux).

14. Catteau Jacques, «Du palais de cristal à l'âge d'or ou les avatars de l'utopie », in Dostoïevski, Paris, L'Herne, 1973, p. 176-195.

15. Cité d'après Catteau, « Du palais de cristal », p. 178.

16. Bucher Lothar, Kulturhistorische Skizzen aus der Industrieausstellung aller Völker, Francfort, 1851, p. 174 (cité d'après Giedion Siegfried, Espace Temps Architecture, I. Lebeer et F.-M. Rosset (trad.), Paris, 1978, p. 163 sq).

17. Ibid.

18. Benjamin Walter, Paris, capitale du XIXe siècle ", in : Gesammelte Schriften, éd. R. Tiedemann, vol. V, 1, Francfort, Suhrkamp, 1982, p. 62 (réédition de l'esquisse française en format poche aux éditions Allia : Paris capitale du XIXème siècle, Paris, Allia, 2003, p. 13).

19. Benjamin, « Paris, capitale du XIX siècle », p. 62 (éd. fr. p. 14). Sur le caractère transitoire, voir aussi les remarques dans le Passagen-Werk: Gesammelte Schriften, vol. V, 1, p. 216 [F 2,9].

20. Cité d'après Kohlmaier Georg, von Sartori Barna, Das Glashaus. Ein Bautypus des 19. Jahrhunderts. Munich, Prestel, 1981, p. 69.

21. Article paru dans Neue Rheinische Zeitung, Politisch-ökonomische Revue, n ${ }^{\circ 5} 5-6$, maioctobre 1850, in Euvres IV. Politique I, M. Rubel (ed), Paris, Gallimard, 1994, p. 397.

22. Benjamin Walter, «Paris, capitale du XIX ${ }^{\mathrm{e}}$ siècle », p. 65 (tr. fr. p. 20-21). Sur cette figure, voir aussi Déotte Jean-Louis, L'Homme de verre. Esthétiques benjaminiennes, Paris, L'Harmattan, 1998.

23. Benjamin Walter, « Paris, capitale du XIX siècle », p. 65 (tr. fr. p. 21).

24. Dostoïevsky Fiodor, Notes d'hiver sur des impressions d'été, W. Bienenstock (trad.), C. Meyer (notes), Paris, L'Entente, 1988, p. 70 sq.

25. Voir à ce propos l'étude étayée de Jacques Catteau, « Du palais de cristal ».

26. Breton André, Nadja, Paris, Gallimard, 1963, p. 18 sq.

27. Benjamin Walter, « Le Surréalisme. Le dernier instant de l'intelligentsia européenne » [1929], M. de Gandillac (trad.) revue par P. Rusch, in Euvres II, Paris, Gallimard, 2000, p. 113-134, p. 118.

28. «Io sogno una casina di cristallo/[...] Una casina come un qualunque mortale/ può possedere,/ che di straordinario non abbia niente,/ ma che sia tutta trasparente,/ di cristallo./ [...] Mi vedrete mangiare, mi potrete vedere/ quando sono a dormire,/ sorprendere i miei sogni;/ mi vedrete fare i miei bisogni,/ mi vedrete quando cambio la camicia ». Palazzeschi, Aldo: « Una casina di cristallo (congedo)»(1913), in Tutte le poesie, A. Dei (ed.), Milan, Mondatori, 2002, p. 316-319. 
29. Scheerbart Paul, L'architecture de verre, P. Galissaire (trad.), Strasbourg, Circé, 1995, p. 29.

30. À ce propos, voir Tafuri Manfredo, Projet et utopie. De l'Avant-garde à la Métropole, F. Brun (trad.), Paris, Bordas, 1979, en particulier p. 102 sqq.

31. Mies van der Rohe, «Bürohaus », in G, $n^{\circ} 1$, Juillet 1923, p. 3. Repris dans Mies van der Rohe, Das kunstlose Wort. Gedanken zur Baukunst, F. Neumeyer (ed.), Munich, Siedler, 1986, p. 8.

32. Benjamin Walter, «Expérience et pauvreté », P. Rusch (trad.), in Euvres II, Paris, Gallimard, 2000, p. 354-372, p. 370.

33. Benjamin Walter, «Brèves ombres », M. de Gandillac (trad.), revue par P. Rusch, in CEuvres II, p. 349-354, p. 352 sq.

34. Benjamin Walter, «Expérience et pauvreté », P. Rusch (trad.), in CEuvres II, Paris, Gallimard, 2000, p. 354-372, p. 369.

35. Ibid.

36. Sur ce point Missac Pierre, "L'architecture de verre ", in Passage de Walter Benjamin, Paris, Seuil, 1987, p. 157-184, en particulier p. 162 sq.

37. Benjamin Walter, «Expérience et pauvreté », p. 369.

38. Benjamin Walter, "Sur Scheerbart ", in Écrits français, Paris, Gallimard, 1991, p. 324-327, p. 326. Ernst Bloch a lui aussi souligné les ambivalences de la vie cristalline dans les utopies architectoniques, voir Bloch Ernst, Le principe espérance. II: Les épures d'un monde meilleur, F. Wuilmart (trad.), Paris, Gallimard, 1982, en particulier p. 346-361.

39. Sennett Richard, "Architectures de verre », in Le temps de la réflexion, VIII, Paris, Gallimard. 1983, p. 125-140, p. 125.

40. Dan Graham, «Glass Buildings: Corporate "Showcases"/ Immeubles de verre: "vitrines" d'entreprises ", in Ma position. Écrits sur mes œuvres, Villeurbanne, Nouveau Musée/Les presses du réel, 1992, p. 144-147, p. 146.

41. Dan Graham, « Glass Buildings », p. 145 sq.

\section{AUTEUR}

\section{EMMANUEL ALLOA}

Docteur en philosophie. Ses travaux ont porté sur la phénoménologie allemande et française, sur Aristote et la question du medium sensible. Parmi ses publications, on peut citer La résistance du sensible. Merleau-Ponty critique de la transparence (Paris, Kimé, 2008) et Nicht(s) sagen.

Strategien der Sprachabwendung im 20. Jahrhundert (transcript, Bielefeld, 2008). 